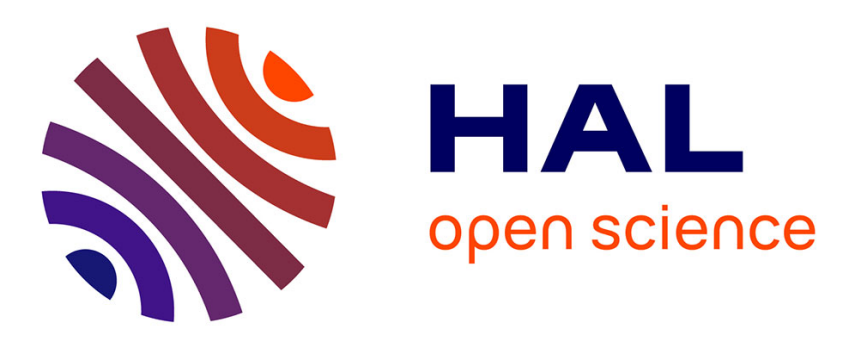

\title{
A New Robust Method for Six-Port Reflectometer Calibration
}

Frank Wiedmann, Bernard Huyart, Eric Bergeault, Louis Jallet

\section{To cite this version:}

Frank Wiedmann, Bernard Huyart, Eric Bergeault, Louis Jallet. A New Robust Method for Six-Port Reflectometer Calibration. IEEE Transactions on Instrumentation and Measurement, 1999, 48 (5), pp.927-931. 10.1109/19.799649 . hal-00351726

\section{HAL Id: hal-00351726 https://hal.science/hal-00351726}

Submitted on 10 Jan 2009

HAL is a multi-disciplinary open access archive for the deposit and dissemination of scientific research documents, whether they are published or not. The documents may come from teaching and research institutions in France or abroad, or from public or private research centers.
L'archive ouverte pluridisciplinaire HAL, est destinée au dépôt et à la diffusion de documents scientifiques de niveau recherche, publiés ou non, émanant des établissements d'enseignement et de recherche français ou étrangers, des laboratoires publics ou privés. 


\title{
A New Robust Method for Six-Port Reflectometer Calibration
}

\author{
Frank Wiedmann, Member, IEEE, Bernard Huyart, Member, IEEE, Eric Bergeault, Member, IEEE, and Louis Jallet
}

\begin{abstract}
A new robust method for finding the parameters of Engen's six-port-to-four-port reduction algorithm for sixport reflectometer calibration has been developed. Like other previously published methods, it uses a minimum of five loads with an unknown but constant absolute value of the reflection coefficient and unknown but well-distributed phases. However, the quality of the parameter estimates is improved, especially in noisy environments, by efficiently eliminating cases in which these earlier methods may become ill-conditioned. The new method has been used successfully to calibrate a newly developed six-port reflectometer in GaAs MMIC technology working between 1.3 GHz and 3.0 GHz.
\end{abstract}

Index Terms-Calibration procedure, network analysis, reflection coefficient measurement, six-port reflectometer, $S$-parameter measurement.

\section{INTRODUCTION}

A MONG the many algorithms which have been proposed for the calibration of six-port reflectometers (SPR's) [1], Engen's six-port-to-four-port reduction [2], [3] seems to be one of the more attractive choices. This procedure determines the dependencies between the different power meter readings, yielding five real-valued reduction parameters which permit to transform the SPR into a virtual four-port. No known standards are required for this reduction. The value measured by the virtual four-port is related to the reflection coefficient of the device under test by a so-called "error box" transformation. The three complex parameters of this transformation may be found by using one of the many existing methods for the calibration of traditional network analyzers.

One of the great advantages of Engen's method is that it makes an efficient use of the redundancy contained in the power meter readings. In fact, the values of the five real parameters of the six-port-to-four-port reduction may be optimized with respect to a nonlinear constraint equation containing the parameter values and the power meter readings. This optimization often permits to improve the accuracy of measurements made with the SPR significantly. However, good initial estimates of the five parameters are needed for the optimization to converge [2].

We will present here a robust method for finding these initial estimates using a minimum of five loads with an unknown but constant absolute value of the reflection coefficient and

Manuscript received July 10, 1996. This work was supported under a grant from the Gottlieb Daimler und Karl Benz-Stiftung.

F. Wiedmann is with Texas Instruments Deutschland $\mathrm{GmbH}$, Freising, Germany,

B. Huyart, E. Bergeault, and L. Jallet are with École Nationale Supérieure des Télé-communications, 75634 Paris, France.

Publisher Item Identifier S 0018-9456(99)06683-8. unknown but well-distributed phases. This method takes its inspiration from an algorithm proposed by Stumper [4], [5] who himself was using an idea of Neumeyer [6]. Stumper's procedure is similar in some aspects to the one presented by Engen in his original paper [2], the latter, however, needed additional loads to determine the five parameters without ambiguity. Our algorithm starts from the same principal ideas as these earlier approaches, but adds several new elements to improve the accuracy in cases where those methods may become ill-conditioned.

Another popular algorithm for finding the initial estimates has also been proposed by Engen [3] and uses nine or more completely unknown but well-distributed loads. This method generally requires a larger number of measurements to be made during calibration than the procedure presented here which only needs a minimum of five loads. It also seems to have some problems in cases where all the reflection coefficients of the unknown loads used for the calibration have approximately the same absolute value [7].

\section{SIX-PORT-TO-FOUR-PORT REDUCTION}

Using the same notation as Stumper [4], the six-port-tofour-port reduction [2] is given by the equations

$$
\begin{aligned}
P_{1} & =|w|^{2} \\
Z P_{2} & =\left|w-w_{1}\right|^{2} \\
R P_{3} & =\left|w-w_{2}\right|^{2}
\end{aligned}
$$

where $w$ is the complex reflection coefficient at the input of the imaginary ideal four-port reflectometer and the $P_{i}=\frac{p_{i}}{p_{4}}$ denote the power values $p_{1}$ to $p_{3}$, measured at the ports labeled 1 to 3 , normalized with respect to the power value $p_{4}$ measured at the reference port 4 of the SPR. The five reduction parameters to be determined by the calibration are the values of the real positive variables $Z, R$, and $w_{1}$, and the real and imaginary parts of the complex variable $w_{2}$.

Engen [2] has shown that the variable $w$ may be eliminated from (1) to (3) yielding the nonlinear constraint equation

$$
\begin{aligned}
A P_{1}^{2} & +B Z^{2} P_{2}^{2}+C R^{2} P_{3}^{2}+(C-A-B) Z P_{1} P_{2} \\
& +(B-C-A) R P_{1} P_{3}+(A-B-C) Z R P_{2} P_{3} \\
& +A(A-B-C) P_{1}+B(B-C-A) Z P_{2} \\
& +C(C-A-B) R P_{3}+A B C=0
\end{aligned}
$$

where

$$
\begin{aligned}
& A=\left|w_{1}-w_{2}\right|^{2} \\
& B=\left|w_{2}\right|^{2}
\end{aligned}
$$




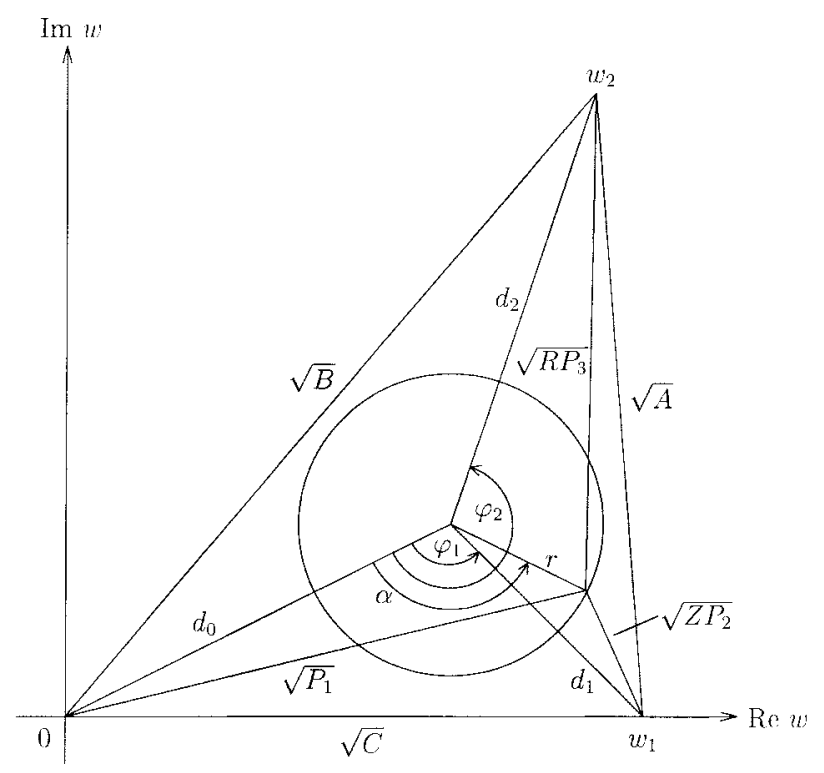

Fig. 1. The $w$ plane with the circle of constant $|\Gamma|$ values.

$$
C=w_{1}^{2}
$$

Equation (4) describes the dependencies between the power values with the help of the reduction parameters and must be fulfilled for any value of $w$.

\section{DETERMination OF THE REDUCTION PARAMETERS}

The algorithm for determining the reduction parameters uses the fact that $w$ and the reflection coefficient $\Gamma$ of the device connected to the measurement port of the SPR are related by a bilinear "error box" transformation of the form

$$
w=\frac{a \Gamma+b}{c \Gamma+1}
$$

which maps circles into circles (with straight lines as limiting cases) [2]. Thus, measurements of loads with a constant absolute value of $\Gamma$ will be lying on a circle in the $w$ plane. However, radius and center of this circle are unknown, since the parameters $a, b$, and $c$ of the bilinear transformation have not yet been determined.

\section{A. Determination of $Z$ and $R$}

This first step of the calibration procedure is very similar to the one proposed by Neumeyer [6] and Stumper [4] with the difference that it adds the possibility to eliminate illconditioned configurations.

Fig. 1 shows an example of the $w$ plane with the circle formed by loads with a constant absolute value of $\Gamma$. The idea is to determine the minima $P_{i \min }$ and the maxima $P_{i \max }$ of the $P_{i}$ corresponding to this circle. This may be done by observing that using the law of cosine, one can write the following equations for the points lying on the circle

$$
\begin{aligned}
P_{1} & =d_{0}^{2}+r^{2}-2 d_{0} r \cos \alpha \\
Z P_{2} & =d_{1}^{2}+r^{2}-2 d_{1} r \cos \left(\alpha-\varphi_{1}\right) \\
R P_{3} & =d_{2}^{2}+r^{2}-2 d_{2} r \cos \left(\alpha-\varphi_{2}\right)
\end{aligned}
$$

where $d_{0}, d_{1}$, and $d_{2}$ are the distances between the circle center and the origin, $w_{1}$, and $w_{2}$, respectively, $r$ is the radius of the circle, $\alpha$ is the angle between the origin and a point on the circle with respect to the circle center, and $\varphi_{1}$ and $\varphi_{2}$ are the angles between the origin and $w_{1}$ and $w_{2}$ with respect to the circle center, respectively.

It is possible to eliminate $\alpha$ from (9) to (11), resulting in three equations of ellipses of the form (cf., [2])

$$
X_{1} P_{i}^{2}+2 X_{2} P_{i} P_{k}+X_{3} P_{k}^{2}+2 X_{4} P_{i}+2 X_{5} P_{k}+1=0
$$

$(i, k=1,2,3 ; i \neq k)$, which are linear in their five parameters $X_{1}$ to $X_{5}$. These parameters may be determined with the help of five loads having a constant absolute value of $\Gamma$ but different phases by solving the corresponding system of linear equations. To obtain more accurate results in the presence of noise, more than five loads may be used to find least-squares solutions of the systems.

Once $X_{1}$ to $X_{5}$ have been found, the extrema of $P_{i}$ may be calculated by (cf., [4])

$$
\begin{aligned}
& P_{\text {iext }} \\
& \quad=\frac{1}{X_{1} X_{3}-X_{2}^{2}}\left(X_{2} X_{5}-X_{3} X_{4}\right. \\
& \left.\quad \pm \sqrt{\left(X_{2} X_{5}-X_{3} X_{4}\right)^{2}-\left(X_{1} X_{3}-X_{2}^{2}\right)\left(X_{3}-X_{5}^{2}\right)}\right)
\end{aligned}
$$

However, we have made the experience that this method tends to become inaccurate for configurations where the corresponding ellipse is relatively flat, since there is always a certain amount of noise in the power measurements. The case of a flat ellipse is the one which is most sensitive to this noise; here, small inaccuracies in the measured power values may lead to rather large errors in the $P_{\text {iext }}$ calculated from (13) via (12). This situation can easily be detected by the fact that the two possible pairs $P_{i}, P_{k}$ and $P_{i}, P_{\ell}(\ell=1,2,3 ; \ell \neq i ; \ell \neq k)$ do not yield approximately the same result for $P_{i}$, but it may be difficult to decide which of these two different results should be used.

To solve this problem, it is useful to obtain a larger number of estimates for the minimum and maximum of $P_{i}$ and to retain the median of them as the final solution in order to eliminate such ill-conditioned situations. Using the trigonometrical relation (cf., [8])

$$
k_{1} \cos \left(\alpha-\theta_{1}\right)+k_{2} \cos \left(\alpha-\theta_{2}\right)=k \cos (\alpha-\theta)
$$

with $^{1}$

$$
\begin{aligned}
& k=\sqrt{k_{1}^{2}+k_{2}^{2}+2 k_{1} k_{2} \cos \left(\theta_{2}-\theta_{1}\right)} \\
& \theta=\arctan \frac{k_{1} \sin \theta_{1}+k_{2} \sin \theta_{2}}{k_{1} \cos \theta_{2}+k_{2} \cos \theta_{2}}
\end{aligned}
$$

it is easy to see that every linear combination of $P_{k}$ and $P_{\ell}$ will be of the same general form as (9) to (11), namely $K_{1}+K_{2} \cos (\alpha-\varphi)$. (Here $K_{1}, K_{2}$, and $\varphi$ are functions of $r, d_{0}, d_{1}, d_{2}, \varphi_{1}, \varphi_{2}, Z, R$, and the coefficients of $P_{k}$

\footnotetext{
${ }^{1}$ In this paper, the notation $\arctan \frac{y}{x}$ is used as an abbreviation for $\operatorname{Im}(\ln (x+j y))$, where $j$ is the imaginary unit with $j^{2}=-1$. This expression is equivalent to the result of the Fortran or $\mathrm{C}$ function $\operatorname{atan} 2(y, x)$.
} 
and $P_{\ell}$ in the linear combination, but are independent of $\alpha$.) Each of these linear combinations will thus as well form an ellipse together with $P_{i}$. Therefore, by substituting various linear combinations of $P_{k}$ and $P_{\ell}$ (like $P_{k}+P_{\ell}, P_{k}-P_{\ell}$, $P_{k}+2 P_{\ell}$, etc.) for $P_{k}$ in (12) and applying (13) to the solution of the corresponding systems of linear equations, the required additional estimates for the extrema of $P_{i}$ can be obtained.

Once the minima and maxima of $P_{1}$ to $P_{3}$ have been found, $Z$ and $R$ may be calculated as in [6] and [4] by noting that from (9) to (11) follows that

$$
\begin{aligned}
\sqrt{P_{1 \max }} \mp \sqrt{P_{1 \min }} & =\sqrt{Z P_{2 \max }} \mp \sqrt{Z P_{2 \min }} \\
& =\sqrt{R P_{3 \max }} \mp \sqrt{R P_{3 \min }} \\
& =2 r
\end{aligned}
$$

which leads to

$$
\begin{aligned}
& Z=\left(\frac{\sqrt{P_{1 \max }} \mp \sqrt{P_{1 \min }}}{\left.\sqrt{P_{2 \max }} \mp \sqrt{P_{2 \min }}\right)^{2}}\right. \\
& R=\left(\frac{\sqrt{P_{1 \max }} \mp \sqrt{P_{1 \min }}}{\sqrt{P_{3 \max }} \mp \sqrt{P_{3 \min }}}\right)^{2} .
\end{aligned}
$$

In these equations, the minus signs have to be used if $d_{i}>r$ for the corresponding $d_{i}$ in (9) to (11), i.e., if the origin, $w_{1}$, and $w_{2}$, respectively, are positioned outside the circle formed by the loads with constant $|\Gamma|$. This will be the case in most situations where the SPR is designed in a way that the socalled $q$-points [1] have an absolute value greater than one and where passive loads are used for calibration.

\section{B. Determination of $A, B$, and $C$}

This second step of the calibration procedure is now completely different from the one proposed by Stumper [4], [5]. In his method, the remaining reduction parameters are determined from the solution of an ellipse equation formed by two different linear combinations of $P_{1}$ to $P_{3}$. Using his algorithm, there is no way to find an accurate solution if this ellipse is very flat and there is noise in the measurements.

It would be an advantage if we were able to obtain not only one estimate like Stumper, but several of them, like it was the case in the previous section, so that we could eliminate such ill-conditioned configurations by using the method described there. This can be done if we find a way to calculate the remaining three reduction parameters from the minima and maxima of linear combinations of $P_{1}$ to $P_{3}$.

Fortunately, there exists a simple solution for this problem. Using (9) to (11) and (14) to (16) as well as the law of cosine, it is possible to show that the following relations hold for the three newly defined quantities $Q_{A}, Q_{B}$, and $Q_{C}$ :

$$
\begin{aligned}
Q_{A}=R P_{3}-Z P_{2} & =d_{2}^{2}-d_{1}^{2}-2 r \sqrt{A} \cos \left(\alpha-\varphi_{A}\right) \\
\varphi_{A} & =\arctan \frac{d_{2} \sin \varphi_{2}-d_{1} \sin \varphi_{1}}{d_{2} \cos \varphi_{2}-d_{1} \cos \varphi_{1}} \\
Q_{B}=P_{1}-R P_{3} & =d_{0}^{2}-d_{2}^{2}-2 r \sqrt{B} \cos \left(\alpha-\varphi_{B}\right) \\
\varphi_{B} & =\arctan \frac{-d_{2} \sin \varphi_{2}}{d_{0}-d_{2} \cos \varphi_{2}} \\
Q_{C}=Z P_{2}-P_{1} & =d_{1}^{2}-d_{0}^{2}-2 r \sqrt{C} \cos \left(\alpha-\varphi_{C}\right)
\end{aligned}
$$

$$
\varphi_{C}=\arctan \frac{d_{1} \sin \varphi_{1}}{d_{1} \cos \varphi_{1}-d_{0}} .
$$

Thus, after the extrema of $Q_{A}, Q_{B}$, and $Q_{C}$ have been determined by the method presented in the previous section ${ }^{2}$, $A, B$, and $C$ may be calculated as

$$
\begin{aligned}
& A=\left(\frac{Q_{A \max }-Q_{A \text { min }}}{4 r}\right)^{2} \\
& B=\left(\frac{Q_{B \max }-Q_{B \text { min }}}{4 r}\right)^{2} \\
& C=\left(\frac{Q_{C \text { max }}-Q_{C \text { min }}}{4 r}\right)^{2}
\end{aligned}
$$

where $r$ follows from (17) as

$$
r=\frac{\sqrt{P_{1 \max }} \mp \sqrt{P_{1 \min }}}{2} .
$$

When $A, B$, and $C$ are known, $w_{1}$ and $w_{2}=u_{2}+j v_{2}(j$ being the imaginary unit with $j^{2}=-1$ ) can be calculated as in [3] as

$$
\begin{aligned}
& w_{1}=\sqrt{C} \\
& u_{2}=\frac{B+C-A}{2 w_{1}} \\
& v_{2}= \pm \sqrt{B-u_{2}^{2}} .
\end{aligned}
$$

The sign in (32) cannot be found without some known loads. It will be determined during the calibration of the "error box." The value $w=u+j v$ of an unknown load is then given by [3]

$$
\begin{aligned}
& u=\frac{P_{1}-Z P_{2}+w_{1}^{2}}{2 w_{1}} \\
& v=\frac{P_{1}-R P_{3}+u_{2}^{2}+v_{2}^{2}-2 u u_{2}}{2 v_{2}} .
\end{aligned}
$$

\section{FINAL STEPS}

After the initial values of $Z, R, w_{1}, u_{2}$, and $v_{2}$ (or of $Z$, $R, A, B$, and $C$ ) have been found, they may be optimized by using either (4) as proposed by Engen in his original paper [2] or with the help of a different constraint equation proposed by Potter and Hjipieris [9], which tends to converge better in the presence of noise. It is also possible to use the method proposed by Judish and Engen in [10], which has some interesting statistical properties for on-line error estimation but usually takes rather longer compared with the other two constraint equations. It optimizes not only the five reduction parameters but also all the $w$ values of the different loads, resulting in a large number of variables which have to be optimized.

Finally, the three complex parameters of the "error box" transformation (8) have to be determined. One simple possibility for this is to use three known loads and to solve the resulting system of linear equations for the three parameters $a, b$, and $c$. Many other, more sophisticated methods have been described, e.g., by Kása [11], who only uses one known load

\footnotetext{
${ }^{2}$ This method may be applied here, because, as mentioned before, linear combinations of the normalized powers like $Q_{A}, Q_{B}$, and $Q_{C}$ are of the same general form as the normalized powers themselves.
} 


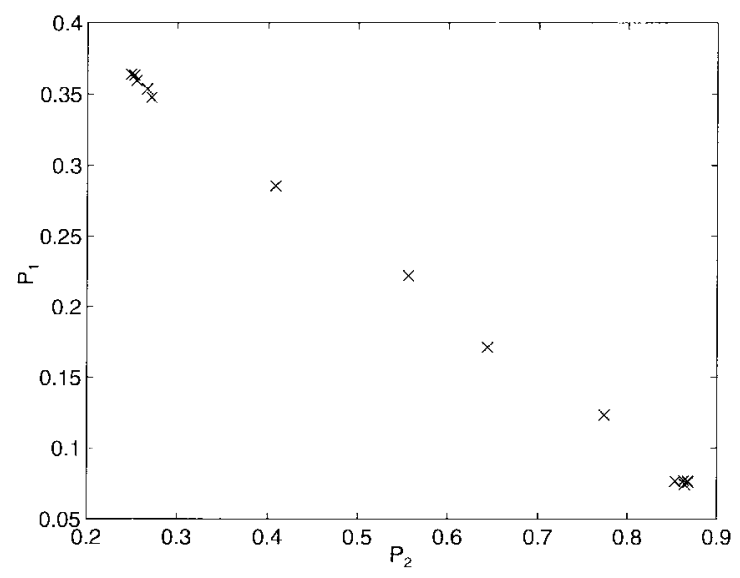

Fig. 2. Plot of $P_{1}$ against $P_{2}$ at $2.5 \mathrm{GHz}$.

together with two different sliding terminations. For a dual SPR, one of the many existing algorithms for calibration of automatic network analyzers may be used, e.g., the popular "Thru-Reflect-Line" [3] or one of its generalizations [12].

The sign in (32) may be determined by comparing the values of the reflection coefficient for an approximately known load obtained after full calibration or by verifying that the reflection coefficients of the loads used to find the initial estimates of the six-port-to-four-port reduction all have the same absolute value and rotate in the correct sense [6]. This method may also be used to determine the signs in (17) to (19), if this should be necessary.

\section{EXPERIMENTAL RESULTS}

The method described here has been used to calibrate a newly developed SPR in GaAs MMIC technology [13], which works between $1.3 \mathrm{GHz}$ and $3.0 \mathrm{GHz}$. The integrated diode detectors were linearized at $2.0 \mathrm{GHz}$ using the method described in [14] with the correction function given in [15].

In the beginning, we had used the method proposed by Stumper [4], [5] to find the initial estimates for Engen's six-port-to-four-port reduction algorithm. This worked well at most frequencies, but there remained problems at some frequency points, especially around $2.5 \mathrm{GHz}$, where it was almost impossible to calibrate the SPR with this method.

A closer examination soon showed the reason for these difficulties. As can be seen in Fig. 2, there was almost a linear relationship between $P_{1}$ and $P_{2}$ so that due to the noise in the measurements, it was practically impossible to determine their minima and maxima accurately from this ellipse which had nearly degraded into a straight line, even when using a large number of loads. (For comparison, Fig. 3 shows a more typical situation with a well-shaped ellipse.) It would, of course, have been possible to get the necessary values from the other two ellipses, but in an automated procedure it may be difficult to decide which of the different results should be trusted. A more severe problem is the last step of the algorithm where $w_{1}$ is calculated from the parameters of the ellipse formed by $P_{1}$ and $Z P_{2}$, which cannot be determined very accurately due to this ill-conditioned configuration.

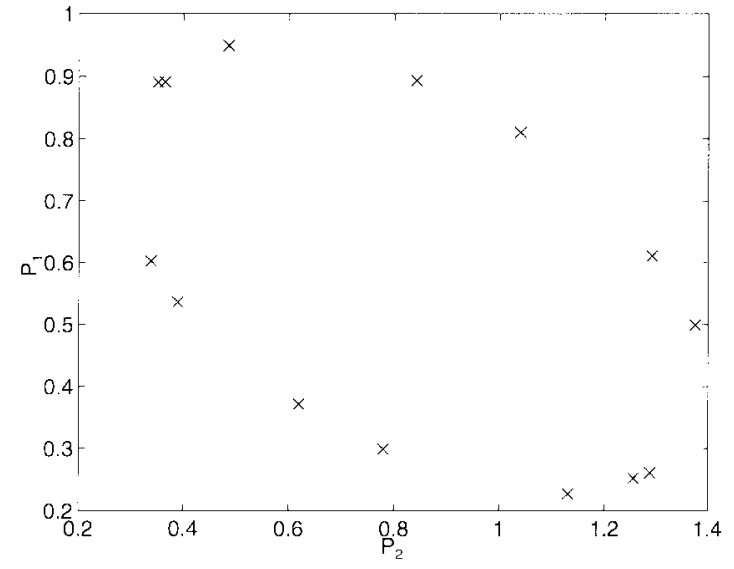

Fig. 3. Plot of $P_{1}$ against $P_{2}$ at $1.8 \mathrm{GHz}$.

These problems were finally solved by the idea to use several linear combinations of the powers as described before in order to obtain more reliable estimates for the minima and maxima of the $P_{i}$ and to extend this method to the second part of the algorithm where $A, B$, and $C$ are determined, resulting in a fairly robust algorithm which is likely to work for almost any SPR configuration.

The SPR in MMIC technology was calibrated with the new method using eight well-distributed loads with $|\Gamma|=0.5$ and eight different combinations of the $P_{i}$ and the $Q_{i}$ for finding the minima and the maxima. The initial estimates were optimized using (4) with a Newton descent algorithm. The virtual four-port was then calibrated using three known loads (open, short, match).

It was found that from the eight different estimates for each of the extrema of the $P_{i}$ and the $Q_{i}$, not more than one or two were totally out of range due to an ill-conditioned configuration. The initial estimates obtained with the new method never differed by more than $7 \%$ from the values obtained after optimization, and there were no cases where the optimization did not converge. The reflection coefficients $\Gamma_{\mathrm{SPR}}$ measured with the new SPR after this calibration were compared to the reflection coefficients $\Gamma_{\mathrm{NA}}$ measured with a commercial network analyzer. The maximum absolute difference $\delta=\left|\Gamma_{\mathrm{SPR}}-\Gamma_{\mathrm{NA}}\right|$ between the measured values ${ }^{3}$ was 0.02 between $1.6 \mathrm{GHz}$ and $2.6 \mathrm{GHz}$ and 0.04 between 1.3 $\mathrm{GHz}$ and $3.0 \mathrm{GHz}^{4}$ for loads distributed over the whole Smith chart [13].

\section{CONCLUSION}

A new robust method for finding the parameters of Engen's six-port-to-four-port reduction algorithm has been developed.

\footnotetext{
${ }^{3}$ The absolute difference between the reflection coefficients is the most meaningful quantity for an SPR, since there are no separate error mechanisms for amplitude and phase errors in this device. Giving a relative difference would not be very useful either, because the accuracy of an SPR generally does not improve very significantly near the center of the Smith chart.

${ }^{4}$ There are two reasons for the larger differences near the band edges: first, the sensitivity of the power detectors is decreased, so that there are larger uncertainties in the power measurements, and second, the so-called $q$-points are in less favorable positions, so that these uncertainties also have a stronger influence on $\Gamma_{\mathrm{SPR}}$. This has nothing to do with the accuracy of the calibration algorithm itself.
} 
It uses a minimum of five loads with an unknown but constant absolute value of the reflection coefficient and unknown but well-distributed phases. The new method improves similar existing algorithms by calculating several estimates for all parameter values which have to be determined, making it possible to eliminate configurations where the corresponding systems of linear equations are ill-conditioned. ${ }^{5}$ Such a situation had occurred at some frequencies during the calibration of a newly developed SPR in GaAs MMIC technology functioning between $1.3 \mathrm{GHz}$ and $3.0 \mathrm{GHz}$. The new method made it possible to overcome these problems and to calibrate the SPR over the whole operating range without any difficulty.

\section{REFERENCES}

[1] G. F. Engen, "The six-port reflectometer: An alternative network analyzer," IEEE Trans. Microwave Theory Tech., vol. MTT-25, pp. 1075-1080, Dec. 1977.

[2] _ "Calibrating the six-port reflectometer by means of sliding terminations," IEEE Trans. Microwave Theory Tech., vol. MTT-26, pp. 951-957, Dec. 1978.

[3] G. F. Engen and C. A. Hoer, “'Thru-reflect-line': An improved technique for calibrating the dual six-port automatic network analyzer," IEEE Trans. Microwave Theory Tech., vol. MTT-27, pp. 987-993, Dec. 1979.

[4] U. Stumper, "Finding initial estimates needed for the Engen method of calibrating single six-port reflectometers," IEEE Trans. Microwave Theory Tech., vol. 38, pp. 946-949, July 1990.

[5] _ , "Sechstorreflektometrie in der PTB-Untersuchung von Kalibrierverfahren," Mikrowellen \& HF Mag., vol. 18, pp. 162-169, 1992.

[6] B. Neumeyer, "A new analytical method for complete six-port reflectometer calibration," IEEE Trans. Instrum. Meas., vol. 39, pp. 376-379, Apr. 1990.

[7] J. Li, R. G. Bosisio, and K. Wu, "Dual-tone calibration of six-port junction and its application to the six-port direct digital millimetric receiver," IEEE Trans. Microwave Theory Tech., vol. 44, pp. 93-99, Jan. 1996

[8] I. N. Bronstein and K. A. Semendjajew, Taschenbuch der Mathe-matik, 23rd ed. Berlin, Germany: Verlag Harri Deutsch, 1987, sect. 2.5.2.1.4, p. 183.

[9] C. M. Potter and G. Hjipieris, "A robust six- to four-port reduction algorithm," in 1993 IEEE MTT-S Int. Microwave Symp. Dig., pp. 1263-1266.

[10] R. M. Judish and G. F. Engen, "On-line accuracy assessment for the dual six-port ANA: Statistical methods for random errors," IEEE Trans. Instrum. Meas., vol. IM-36, pp. 507-513, June 1987.

[11] I. Kása, "Closed-form mathematical solutions to some network analyzer calibration equations," IEEE Trans. Instrum. Meas., vol. IM-23, pp. 399-402, Dec. 1974.

[12] H.-J. Eul and B. Schiek, "A generalized theory and new calibration procedures for network analyzer self-calibration," IEEE Trans. Microwave Theory Tech., vol. 39, pp. 724-731, Apr. 1991.

[13] F. Wiedmann, B. Huyart, E. Bergeault, and L. Jallet, "New structure for a six-port reflectometer in monolithic microwave integrated-circuit technology," IEEE Trans. Instrum. Meas., vol. 46, pp. 527-530, Apr. 1997.

[14] E. Bergeault, B. Huyart, G. Geneves, and L. Jallet, "Characterization of diode detectors used in six-port reflectometers," IEEE Trans. Instrum. Meas., vol. 40, pp. 1041-1043, Dec. 1991.

${ }^{5}$ The algorithm is also very useful to recognize if there is something wrong with the SPR system: if the ellipses used for determining $Z$ and $R$ are looking strange, there is either a problem with the power detectors (bad linearity or repeatability), or the SPR is not really a completely linear circuit like it is required by SPR theory [1].
[15] C. Potter and A. Bullock, "Nonlinearity correction of microwave diode detectors using a repeatable attenuation step," Microwave J., vol. 36, pp. 272-279, May 1993.

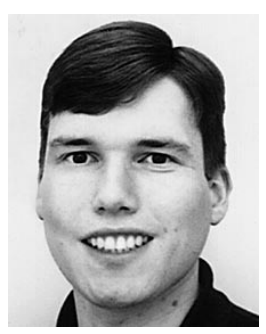

Frank Wiedmann (M'98) was born in Stuttgart, Germany, in 1968. He received the Dipl.-Ing. degree in electrical engineering from the University of Stuttgart in 1994 and the Ph.D. degree in electronics and communications from the École Nationale Supérieure des Télécommunications, Paris, France, in 1997, where he worked on the development of new structures for six-port reflectometers, on algorithms for their calibration, and on the design of monolithic microwave integrated circuits.

He is now with Texas Instruments Deutschland $\mathrm{GmbH}$, Freising, Germany, where he is working on the design of radiofrequency integrated circuits.

Bernard Huyart (M'92) was born in France in 1954. He received the Ingénieur degree in electrical engineering from the École Universitaire des Ingénieurs de Lille, Paris, France, in 1977, the Ph.D. degree in physics from the École Nationale Supérieure des Télécommunications (ENST), Paris, in 1986, and the research habilitation degree from the University of Limoges, Limoges, France, in 1995.

In 1978, he joined the staff of ENST, where he is currently a Professor. His research interests include microwave instrumentation (six-port techniques, electrooptic probing), noise and nonlinear device measurement and modelization, and the design of monolithic microwave integrated circuits (MMIC's).

Eric Bergeault (M'96) was born in France in 1963. He received the Diplôme d'Études Approfondies (DEA) degree from the University of Limoges, Limoges, France, in 1987, and the Ph.D. degree in electronics and communications from the École Nationale Supérieure des Télécommunications (ENST), Paris, France, in 1991.

From 1987 to 1990, he was a Research Engineer with the Laboratoire Central des Industries Électriques (LCIE), Fontenay aux Roses, France. He joined the staff of ENST in 1991, where he is currently an Assistant Professor. His research interest is in the field of microwave instrumentation and he is mainly involved in research with the six-port network analyzer, characterization of nonlinear devices, and applications to the optimization of power amplifiers.

Dr. Bergeault is a member of the Editorial Review Committee of the IEEE Transactions on Instrumentation and MEasurement.

Louis Jallet was born in France in 1946. He is a graduate of the National Institute for Telecommunications Administrations, Paris, France.

Since 1975, he has worked in the Department of Electronics and Physics, École Nationale Supérieure des Télécommunications (ENST), Paris, then in the Communications Department as Head of the Radio-Frequency and Microwave Group, which is associated with the Centre National de la Recherche Scientifique. His research activities primarily concern microwave instrumentation. He has published many papers about six-port junction systems and MMIC functions. 